\title{
Anelli Henseliani topologici - II $\left(^{*}\right)$.
}

\author{
Paolo Valabrega (a Torino) $\left(^{* *}\right)$
}

Summary. - In the present work we study some problems about henselian triples and henselization which have been introduced in our article [18]. Mainly we prove that henselian triples coincide with strong henselian triples and give a new formulation of the Hensel lemma, stronger than that we gave in [18]. Then we investigate some properties of henselian triples (change. ment of ideal or of topology, ecc.) and prove commutativity with quotient.

\section{Introduxione.}

Il presente lavoro è il diretto proseguimento dell'articolo [18], in cui abbiamo introdotto $i$ concetti relativi alle terne henseliane e alla henselizzazione delle terne.

Precisamente, nel precedente lavoro abbiamo introdotto i due concetti di terna henseliana e di terna fortemente henseliana $([18]$, n. 2) ed i risultati ottenuti ci hanno permesso di costruire l'henselizzazione (non forte) nella categoria delle terne con base numerabile per gli intorni dello 0 .

In [18] restava aperto il problema di stabilire l'equivalenza fra le due definizioni di $H$-terna ovvero della costruzione di una eventuale henselizzazione forte.

In questo articolo dimostriamo che tutte le terne henseliane sono forti, sotto condizioni di numerabilità per gli intorni dello 0 .

Resta percio chiarito che i risultati ottenuti in [18] riguardano proprio le terne che soddisfano alla tesi del lemma di Hensel versione Bourbakr.

Fra i risultati più notevoli del presente lavoro, oltre a quello ora indicato, e'è una versione del lemma di HeNSEL ancora più forte di quella data in [18] (teorema 5) e una caratterizzazione di tutte le possibili topologie che, introdotte in una coppia henseliana, la rendono una $H$-terna.

Il lavoro è diviso in tre sezioni.

Nel n. 1 riprendiamo brevemente i risultati ottenuti in [18], esponendo i problemi lasciati aperti.

Nel n. 2 dimostriamo l'equivalenza fra le due definizioni di terna henseliana ed enunciamo la nuova versione del lemma di HerseL.

Nel n. 3 infine dimostriamo alcune proprietà delle terne henseliane e dell'henselizzazione, analoghe a proprietà delle coppie (rimpicciolimento dellideale, passaggio al quoziente, ecc.).

(*) Lavoro eseguito nell'ambito dei contratti di ricerca del Comitato Nazionale per la Matematica del C.N.R.

(**) Entrata in Redazione il 27 maggio 1971 .

20 - Annali di Matematica 
1. - In questo numero richiamiamo le principali proprietà relative alle coppie $e$ alle terne henseliane $\mathrm{e}$ i concetti riguardanti l'henselizzazione, sia per le coppie ([11]), sia per le terne ([18]).

Una coppia $(A, \mathfrak{m})$ è costituita da un anello commutativo unitario e da un suo ideale qualsiasi $\mathfrak{m}$. Un omomorfismo di coppie $f:(A, \mathfrak{m}) \rightarrow\left(A^{\prime}, \mathfrak{m}^{\prime}\right)$ è un omomorfismo di anelli $f: A \rightarrow A^{\prime}$, tale che $f(\mathfrak{m}) \subseteq \mathfrak{m}^{\prime}$.

Una coppia $(A, \mathfrak{m})$ si dice henseliana o $H$-coppia se e solo se soddisfa alla tesi del lemma di HeNsel versione BouRBaKI ([5], cap. III, $\S 4$, n. 3, teorema 1), per i polinomi unitari. È noto che, in una coppia henseliana, l'ideale è sempre contenuto nel radicale ([13], proposizione 1$)$.

Data una coppia $(A, \mathfrak{m})$, si può associare a questa la sua henselizzazione, che è una coppia henseliana ${ }^{h}(A, \mathfrak{m})=\left({ }^{h} A,{ }^{h} \mathfrak{m}\right)$, insieme con un morfismo di coppie $i:(A, \mathfrak{m}) \rightarrow^{n}(A, \mathfrak{m})$, tale che sia soddisfatta la seguente proprietà universale: se $\varphi:(A, \mathfrak{m}) \rightarrow\left(A^{\prime}, \mathfrak{m}^{\prime}\right)$ è un morfismo in una coppia henseliana, esiste uno e un solo morfismo ${ }^{h} \varphi:\left({ }^{n} A,{ }^{h} \mathfrak{m}\right) \rightarrow\left(A^{\prime}, \mathfrak{m}^{\prime}\right)$ tale che si abbia: $\varphi={ }^{h} \varphi \circ i$. In [11] è dimostrata l'esistenza per l'henselizzazione delle coppie.

In [18] si è introdotto il concetto di terna $(A, \mathfrak{m}, \tau)$ formata da un anello commutativo unitario, da una topologia lineare separata $\tau$ sopra $A$ e da un ideale $\mathfrak{m}$ chiuso per la topologia e contenuto nel radicale di $A$ (in [18], n. 5 abbiamo anche trattato il caso in cui $m$ non sia contenuto nel radicale, riconducendolo al caso precedente).

Un morfismo di terne $f:(A, \mathfrak{n}, \tau) \rightarrow\left(A^{\prime}, \mathfrak{m}^{\prime}, \tau^{\prime}\right)$ è un omomorfismo di anelli $f: A \rightarrow A^{\prime}$, continuo e tale che $f(\mathfrak{m}) \subseteq \mathfrak{m}^{\prime}$.

In [18] si è esteso il concetto di coppia henseliana introducendo quelli di terna henseliana e di terna fortemente henseliana:

Definizione 1. - La terna $(A, \mathfrak{m}, \tau)$ si dice henseliana (o $H$-terna) se e solo se sono soddisfatte le seguenti condizioni:

1) $\mathfrak{m} \subseteq \operatorname{Rad}(A)$;

2) per ogni serie ristretta ([5], cap. III, $\S 4$, n. 2, definizione 2) $R \in A\{X\}$, la cui immagine canonica in $(A / \mathfrak{m})\{X\}$ è prodotto di un polinomio unitario $\bar{P}$ e di una serie ristretta $\bar{Q}$ fra loro coprimi, esiste una e una sola coppia $(P, Q)$ formata da un polinomio unitario $P$ e da una serie ristretta $Q \in A\{X\}$, tale che $R=P Q$ e che le immagini canoniche di $P$ e di $Q$ in $(A / \mathfrak{m})\{X\}$, siano rispettivamente $\bar{P}$ e $\bar{Q}$. Inoltre, se $R$ è un polinomio, allora anche $Q$ è un polinomio.

DeFinIzIone $1^{\prime}$. - La terna $(A, \mathfrak{m}, \tau)$ si dice fortemente henseliana (o $H$-terna forte) se e solo se è una $H$-terna e il polinomio unitario e la serie ristretta di cui alla Definizione 1 sono coprimi in $A\{X\}$.

In modo equivalente ma più espressivo possiamo dire che una terna ̀̀ fortemente henseliana se e solo se soddisfa alla tesi del lemma di HEXSEL versione BouRBAKI ([5], cap. III, $\$ 4$, n. 3 , teorema 1 ). 
Nel n. 2 del presente lavoro vedremo che le due definizioni sono equivalenti, sotto l'ipotesi di numerabilità per gli intorni dello 0 di $A$, risolvendo così uno dei principali problemi rimasti aperti in [18], dove si era vista l'equivalenza solo per certi tipi di terne (ideale con elementi topologicamente nilpotenti, anello base completo).

In [18] viene anche costruita l'henselizzazione per la categoria delle terne con base numerabile degli intorni di 0 . La proprietà universale di cui l'henselizzazione delle terne è soluzione è ovviamente perfettamente analoga a quella delle coppie ([18], n. 5, definizione 4 e teorema 7$)$.

2. - In [18] restava aperto il problema dell'equivalenza fra la definizione di terna henseliana e quella di terna fortemente henseliana.

Ci proponiamo in questo numero di far vedere che una terna henseliana soddisfa sempre alla tesi del lemma di HENSEL versione BouRBAKI, sotto la condizione che gli intorni di 0 abbiano una base numerabile.

Per arrivare alla dimostrazione del nostro teorema abbiamo bisogno di premettere un risultato sugli anelli topologici separati completi:

Proposizione 1. - Sia $A$ un anello topologico separato completo rispetto alla topologia lineare $\tau$, e sia $(P, Q)$ una coppia formata da un polinomio unitario $P$ e da una serie ristretta $Q \in A\{X\}$, coprimi in $A\{X\}$. Allora esiste una e una sola coppia $(B, C)$, formata da una serie ristretta $B$ e da un polinomio $C$ di grado inferiore a quello $d i P$, tale the si abbia: $B P+C Q=1$.

Dimostrazione. - Sia $\left\{\mathfrak{a}_{i}\right\}_{i \in I}$ una base per gli intorni dello 0 di $A$ formata di ideali e $A_{i}=A / \mathfrak{a}_{i}$, per ogni $i \in I$. Le immagini canoniche $P_{i}$ e $Q_{i}$ di $P$ e $Q$ in $A_{i}$ sono polinomi coprimi di $A_{i}[X]$ (si ricordi che tutti gli anelli $A_{i}$ sono discreti e quindi le serie ristrette su $A_{i}$ sono i polinomi). Inoltre $P_{i}$ è unitario; perciò è noto ([5], cap. III, $\S 4$, n. 1, proposizione 1) che esiste una e una sola coppia $\left(B_{i}, C_{i}\right)$ formata da due polinomi in $A_{i}[X]$, con $O_{i}$ di grado inferiore a quello di $P_{i}$ (cioè di $P$ ), tale che si abbia: $B_{i} P_{i}+C_{i} Q_{i}=1$. Le due famiglie $\left(B_{i}\right)_{i \in I}$ e $\left(C_{i}\right)_{i \in I}$ sono coerenti, per l'unicità della coppia $\left(B_{i}, C_{i}\right)$ al variare dell'indice; perciò sono elementi dell'anello $\underset{\leftarrow}{\lim } A_{i}[X]=A\{X\}$ (si ricordi che $A$ è separato completo: [5], §4, n. 2, proposizione 3 ). Posto $B=\left(B_{i}\right)_{i \in I}$ e $O=\left(C_{i}\right)_{i \in I}$, si ha ovviamente in $A\{X\}: B P+C Q=1$ e $C$ ha grado inferiore a quello di $P$.

Sia poi $\left(B^{\prime}, C^{\prime}\right)$ una qualsiasi coppia formata da un polinomio $C^{\prime}$ di grado minore di quello di $P$ e da una serie ristretta $B^{\prime}$, tale che si abbia: $B^{\prime} P+C^{\prime} Q=1$. Proiettando sugli anelli $A_{i}[X]$ si ottiene: $B_{i}^{\prime} P_{i}+C_{i}^{\prime} Q_{i}=1$, per ogni $i \in I$. Ciò significa che $B_{i}^{\prime}=B_{i}, C_{i}^{\prime}=C_{i}$, per ogni $i \in I$, ossia $B=B^{\prime}, O=C^{\prime}$.

A questo punto, salvo avviso in contrario, ci mettiamo sempre in condizioni di numerabilità, cioè supponiamo che gli intorni $d i$ abbiano una base numerabile, in tutti gli anelli linearmente topologizzati che prenderemo in considerazione nel presente lavoro. 
LEMMA. - Sia A un anello linearmente topologizzato separato con base numerabile per gli intorni di $0, \mathfrak{m}$ un suo ideale chiuso. Allora l'applicazione canonica: $A\{X\} \rightarrow$ $\rightarrow(A / \mathfrak{m})\{X\}$ è suriettiva.

Dnostrazione. - Sia $\left\{\mathfrak{a}_{n}\right\}$ una base numerabile di intorni dello 0 di $A$, formata di ideali e totalmente ordinata per inclusione. E noto che una base per gli intorni di 0 in $A / \mathfrak{m}$ è formata dagli ideali $\overline{\mathfrak{a}}_{n}$, immagini canoniche degli ideali $\mathfrak{a}_{n}$ in $A / \mathfrak{m}$ ([8], cap. III, §2, n. 6, proposizione 18).

Sia $\bar{R}=\bar{a}_{0}+\bar{a}_{1} X+\ldots+\bar{a}_{n} X^{n}+\ldots$ una serie ristretta in $(A / \mathfrak{m})\{X\}$. Ciò significa che, per ogni intero $k>0$, esiste un intero $n_{k}$ (supponiamo che sia il minimo) tale che tutti $i$ coefficienti di $\bar{R}$ con indice $n>n_{k}$ stanno in $\overline{\mathfrak{a}}_{k}$. Otteniamo così una successione non decrescente di interi positivi $\left\{n_{k}\right\}$.

Scegliamo ora a piacere $a_{n}$ in $\bar{a}_{n}$, quando $n \leqslant n_{1}$; quando invece $n$ è compreso fra $n_{1}$ ed $n_{2}$, scegliamo in $\bar{a}_{n}$ un rappresentante $a_{n} \in \mathfrak{a}_{1}$, eec.

E chiaro che la serie $R=a_{0}+a_{1} X+\ldots$ ha immagine canoniea $\bar{R}$ ed è ristretta, cioè è una controimmagine di $\bar{R}$ nell'applicazione: $A\{X\} \rightarrow(A / \mathfrak{m})\{X\}$.

Possiamo ora enunciare il seguente

Teorema 1. - Sia $(A, \mathrm{~m}, \tau)$ una terna con ideale separato completo rispetto alla topologia lineare $\tau$, dotata di una base numerabile di intorni di 0 , e siano $P$ un polinomio unitario $e Q$ una serie ristretta a coefficienti in $A$ tali che le loro immagini canoniche $\bar{P}$ e $\bar{Q}$ in $(A / \mathfrak{M})\{X\}$ siano eoprime. Allora condizione necessaria e sufficiente affinchè $P$ e $Q$ siano coprimi in $A\{X\}$ è che essi siano coprimi in $\hat{A}\{X\}(\hat{A}=$ separato completato di $A$ rispetto a $\tau$ ).

Dimostraztone. - La sufficienza della condizione è ovvia, in quanto $A\{X\}$ è un sottoanello di $\hat{A}\{X\}$.

Viceversa, siano $P$ e $Q$ coprimi in $\hat{A}\{X\}$ e $\bar{P}$ e $\bar{Q}$ in $(A / \mathfrak{m})\{X\}$. Ciò significa che esistono due serie ristrette $\bar{R}$ ed $\bar{S}$ a coefficienti in $A / \mathfrak{m}$ tali che si abbia: $\bar{R} \bar{P}+\bar{S} \bar{Q}=1$. Del pari esistono due serie ristrette $B$ e $C$ a coefficienti in $\hat{A}$ tali che si abbia: $B P+C Q=1$. La Proposizione 1 ei permette di supporre che $C$ sia un polinomio di grado inferiore a quello di $P$.

La relazione: $B P+C Q=1$, proiettata sopra $(\hat{A} / \mathfrak{m})\{X\}$ dà: $\bar{B} \bar{P}+\bar{C} \bar{Q}=1$. È chiaro che $(\bar{B}, \bar{C})$ è l'unica coppia di elementi dell'anello $\hat{A} / \mathfrak{m}$ che soddisfi alla condizione indicata nella proposizione 1 .

Osserviamo ora che $\hat{A} / \mathrm{m}$ ì separato completo e coincide anzi, a meno di isomorfismi di anelli topologici, con il separato completato di $A / \mathfrak{m}([9], \S 3$, n. 1 , corollario 1 alla proposizione 4). Quindi esiste in $(\hat{A} / \mathfrak{m})\{X\}$ una e una sola coppia $(\bar{q}, \bar{r})$, formata da una serie ristretta $\bar{q}$ e da un polinomio $\bar{r}$ di grado inferiore a quello di $\bar{P}$ tale che si abbia: $\bar{S}=\bar{q} \bar{P}+\bar{r}$ ([17], teorema 11). Sostituiamo nell'eguaglianza: $\bar{R} \bar{P}+\bar{S} \bar{Q}=1$, ottenendo: $\bar{R} \bar{P}+(\bar{q} \bar{P}+\bar{r}) \bar{Q}=1$. Poichè $\bar{r}$ è un polinomio di grado inferiore a quello di $\bar{P}$, necessariamente si deve avere: $\bar{R}+\bar{q} \bar{Q}=\bar{B}, \bar{r}=\bar{C}$, per l'unicità della coppia $(\bar{B}, \bar{C})$.

Si deduce allora che le due seguenti serie ristrette: $\bar{B}-\bar{q} \bar{Q}=\bar{R}$ e $\bar{C}+\bar{q} \bar{P}=\bar{S}$ 
hanno tutti i coefficienti in $A / \mathrm{m}$. Scegliamo allora una qualsiasi serie ristretta $q$ in $\hat{A}\{X\}$ che abbia immagine canonica coincidente con $\bar{q}$ ( $q$ esiste per il lemma precedente). Allora $B-q Q$ e $C-q P$ sono serie ristrette a coefficienti in $A$, in quanto le loro immagini canoniche hanno i coefficienti in $A / \mathrm{m}$.

Da questo fatto segue immediatamente che $P$ e $Q$ sono coprimi in $A\{X\}$. In effetti si ha: $(B-q Q) P+(C+q P) Q=B P+C Q=1$.

Osservazione. - Siano $A$ un anello linearmente topologizzato separato completo, $P$ un polinomio unitario e $Q$ una serie ristretta coprimi in $A\{X\}$. La proposizione 1 mostra che $P$ e $Q$ si comportano, per certi aspetti, come un polinomio unitario $P^{\prime}$ e un polinomio arbitrario $Q^{\prime}$ nell'anello dei polinomi $A[X]$. In effetti, in $A[X]$, esiste una e una sola coppia $\left(B^{\prime}, C^{\prime}\right)$ di polinomi tale che $C^{\prime}$ abbia grado inferiore a quello di $P^{\prime}$ e che si abbia: $B^{\prime} P^{\prime}+C^{\prime} Q^{\prime}=1$ ([5], cap. III, $\S 4$, n. 1, proposizione 1); del pari, in $A\{X\}$, esiste una e una sola coppia $(B, C)$ formata da una serie ristretta $B$ e da un polinomio $C$ di grado inferiore a quello di $P$ tale che si abbia: $B P+O Q=1$ (proposizione 1).

Questa analogia cade quando l'anello $A$ è separato ma non completo. Cioè è possibile trovare un polinomio unitario $P$ e una serie ristretta $Q$ coprimi in $A\{X\}$, senza che si possa associare alla coppia $(P, Q)$ una coppia $(B, C)$ soddisfacente alla condizione indicata nella proposizione 1 .

Sia, in effetti, $A$ separato ma non completo rispetto alla topologia lineare $\tau$, supposta dotata di una base numerabile per gli intorni di 0 . Sia poi $f$ un elemento qualsiasi di $\hat{A}$, non appartenente ad $A$ ed invertibile in $\hat{A}$ e poniamo $g=1 / f \in \hat{A}$. Si puó certo sempre scrivere: $g=\sum_{0}^{\infty} a_{n}, f=\sum_{0}^{\infty} b_{n}$, con $\lim _{n \rightarrow \infty} a_{n}=\lim _{n \rightarrow \infty} b_{n}=0$. Posto: $D_{n}=a_{0} b_{0}+\left(a_{0} b_{1}+a_{1} b_{0}\right)+\cdots+\left(a_{n} b_{0}+a_{n-1} b_{1}+\ldots+a_{0} b_{n}\right)$, si ha evidentemente: $\lim _{n \rightarrow \infty} D_{n}=f g=1$.

Consideriamo ora la coppia $(P, Q)=\left(X-1, \sum_{0}^{\infty} a_{n} X^{n}\right)$ e facciamo vedere che $P$ e $Q$ sono coprimi in $A\{X\}$. In effetti ciò significa che esistono due serie ristrette $R$ ed $S$ tali che si abbia: $R P+S Q=1$.

Scegliamo $S=\sum_{0}^{\infty} b_{n} X^{n}$ e cerchiamo di determinare $R=\sum_{0}^{\infty} c_{n} X^{n}$. Dalla eguaglianza: $\left(\sum_{0}^{\infty} c_{n} X^{n}\right)\left(X^{0}-1\right)+\left(\sum_{0}^{\infty} b_{n} X^{n}\right)\left(\sum_{0}^{\infty} a_{n} X^{n}\right)=1$, si deduce che si deve avere: $c_{0}=a_{0} b_{0}-1, c_{n}=D_{n}-1$, per $n \geqslant 1$.

Si ha: $\lim _{n \rightarrow \infty} c_{n}=\lim _{n \rightarrow \infty}\left(D_{n}-1\right)=0$, quindi $R=\sum_{0}^{\infty} c_{n} X^{n}$ è ristretta e risolve il problema.

Segue dai precedenti calcoli che $X-1$ e $\sum_{0}^{\infty} a_{n} X_{*}^{n}$ sono coprimi in $A\{X\}$ e quindi in $\hat{A}\{X\}$. Allora devono esistere in $\hat{A}\{X\}$ una serie ristretta $B$ e un polinomio $C$ di grado zero tali che si abbia: $B(X-1)+C\left(\sum_{0}^{\infty} a_{n} X^{n}\right)=1$.

Ponendo $X=1$ (in $\hat{A}$ ha ovviamente senso) si ottiene: $O\left(\sum_{0}^{\infty} a_{n}\right)=1$.
Ne segue che $C=1 / g=f \in \hat{A}-A$. 
Ad esempio si consideri l'anello $A=K[X]$ (ove $K$ è un campo di caratteristica 0$)$ e $\tau$ = topologia $X$-adica. Ovviamente $A$ è separato ma non completo per $\tau$ e si ha: $\hat{A}=K[[X]]$.

Detta $T$ una indeterminata, siano $P=T-1, Q=\exp (X T)=1+X T+\ldots+$ $+(1 / n !) X^{n} T^{n}+\ldots$, non è difficile vedere che, in questo caso, si ha: $C=\exp (-X) \notin K[X]$.

A questo punto siamo in grado di dimostrare l'equivalenza fra le definizioni 1 e $1^{\prime}$ :

TEonena 2. - Sia $(A, \mathrm{~m}, \tau)$ una terna henseliana con una base numerabile di intorni dello 0 . Allora $(A, \mathfrak{m}, \tau)$ è fortemente hensetiana.

Drmostrazione. - Ricordiamo, in primo luogo, che $\mathfrak{m}$ è separato completo per la topologia indotta su di esso da $\tau$ ([18], proposizione 2 ).

Inoltre $(A, \mathfrak{m}, \tau) \hat{~}=(\hat{A}, \mathfrak{m}, \hat{\tau})$ è certamente fortemente henseliana perchè $\hat{A}$ è separato completo ([18], teorema 5). Siano allora $P$ un polinomio unitario e $Q$ una serie ristretta a coefficienti in $A$ tali che si abbia: $(\bar{P}, \bar{Q})=(1)$ in $(A / \mathrm{mr})\{X\}$ ed anche in $(\hat{A} / \mathfrak{m})\{X\}$, in quanto $A / \mathfrak{m}$ è un sottoanello topologico di $\hat{A} / \hat{\mathfrak{m}}=(A / \mathfrak{m})^{\hat{n}}=\hat{A} / \mathfrak{m}$ ([8], cap. III, $\S 2$, n. 7, proposizioni 20 e 21). Ne deduciamo che $P$ e $Q$ sono coprimi in $\widehat{A}\{X\}$ e quindi in $A\{X\}$, in conseguenza del Teorema 1 .

$\mathrm{E}$ del tutto naturale, nelle presenti condizioni, eliminare la doppia terminologia introdotta in [18] (terna henseliana e terna fortemente henseliana) per adottare un termine unico per le terne (con base numerabile di intorni di 0 ) soddisfacenti alla tesi del lemma di Hensel versione BoURBaJI), che chiameremo in tutto il presente lavoro terne henseliane (o $H$-terne).

Vogliamo ora riformulare il lemma di Hensel versione BouRBaki in maniera ancora più forte di quella proposta in [18], teorema 5 (lemma di HENSEL forte), eliminando, oltre all'ipotesi che l'ideale sia formato di elementi topologicamente nilpotenti, anche la condizione di completezza dell'anello base.

Precisamente vale il seguente

TEorema 3. - Sia $(A, \mathfrak{m}, \tau)$ una terna tale che $A$ sia separato, $\mathfrak{n t}$ completo e $\tau$ sia dotata di una base numerabile per gli intorni dello 0. Allora le seguenti condizioni sono equivalenti:

1) $(A, \mathfrak{m})$ è una coppia hensetiana;

2) $(A, \mathfrak{i t}, \tau)$ è una terna henseliana;

3) esiste una base $\left\{a_{n}\right\}_{n \in A}$ per gli intorni dello 0 di $A$ in $\tau$, formata di ideali, tale che tutte te coppie $\left(A / \mathfrak{a}_{n}, \mathfrak{m}\left(A / \mathfrak{a}_{n}\right)\right)$ sono henseliane;

4) per ogni ideale aperto a di $A$, la coppia $(A / \mathfrak{a}, \mathfrak{m}(A / \mathfrak{a}))$ è henseliana;

5) se $R=a_{0}+a_{1} X+\ldots+a_{n} X^{n}+\ldots$ è una serie ristretta a coefficienti in $A$ tale che $a_{0} \in \mathfrak{m}, a_{1}$ è invertibile modulo $\mathfrak{m}$, allora $R$ ammette una e una sola radice in $\mathfrak{m}$; inoltre $\mathrm{th}$ è contenuto nel radicale di $A$. 
Drmostrazione. - Seguiremo il seguente schema di dimostrazione:

$$
\text { 1) } \Rightarrow 4) \Rightarrow 3 \text { ) }(2) \Rightarrow 5 \text { ) } \Rightarrow 1 \text { ) }
$$

a) 1) $\Rightarrow 4)$ : discende immediatamente dal fatto che l'henselizzazione delle coppie commuta con il passaggio al quoziente ([18], corollario 1 al teorema 1 ).

b) 4) $\Rightarrow 3$ ): ovvio.

e) 3) $\Rightarrow 2)$ : la condizione 3) dice che $(A, \mathfrak{m}, \tau)^{\wedge}=(\hat{A}, \mathfrak{m}, \widehat{\tau})$ è una terna henseliana ([18], teorema 5 ), in quanto si: $A / \mathfrak{a}_{n}=\hat{A} / \hat{\mathfrak{a}}_{n}$, per ogni $n \in N$ ([8], cap. III, $\S 2$, n. 7 , proposizione 21).

d) 2) $\Rightarrow 5)$ : l'immagine canonica di $R$ in $(A / \mathfrak{m})\{X\}$ è spezzata nel prodotto del polinomio unitario $X$ e della serie ristretta $\bar{Q}=\bar{a}_{1}+\ldots+\bar{a}_{n} X^{n}+\ldots$, palesemente coprimi in $(A / \mathfrak{m})\{X\}$. Perciò esiste una e una sola coppia $(P, Q)$, formata da un polinomio unitario $P=X-m$ (con $m \in \mathfrak{m}$ ) e da una serie ristretta $Q$ coprimi in $A\{X\}$, tale che $R=P Q$. Ma questo significa che $R(m)=0$, cioè $X=m$ ̀̀ radice di $R$ (si osservi che $R(m)$ ha senso in $\hat{A}$ ).

Supponiamo che $R$ abbia una seconda radice $m^{\prime} \in \mathfrak{m}$. Dividiamo $R$ per il polinomio unitario $X-m^{\prime}$ in $\hat{A}\{X\}\left([17]\right.$, teorema 11): $R=\left(X-m^{\prime}\right) Q^{\prime}+r$, dove $r$ è un polinomio di grado 0 e coincide con l'elemento $R\left(m^{\prime}\right)=0$ di $\hat{A}$.

Dunque $R=\left(X-m^{\prime}\right) Q^{\prime}$, il che è in contraddizione con il rimontamento unico. Infine $\mathfrak{m} \subseteq \operatorname{Rad}(A)$ in quanto $(A, \mathfrak{m}, \tau)$ è una terna henseliana.

e) 5) $\Rightarrow$ 1): la condizione 5) è verificata in particolare quando $R$ sia un polinomio unitario; e questo fatto, insieme con l'ipotesi che sia contenuto nel radicale, implica che $(A, \mathfrak{m})$ sia una coppia henseliana ([11], definizione 1.3 e teorema 5.11).

OSSERVAzione. - Il precedente teorema 3 generalizza il lemma di HENsEL versione BoURBaKT (in condizioni di numerabilità) in modo più forte del teorema 5 che abbiamo dato in [18], in quanto facciamo a meno dell'ipotesi di completezza dell'anello base.

La nostra ipotesi di completezza per l'ideale è poi in accordo con le ipotesi di BOURBAKI, in quanto un ideale chiuso in un anello separato completo è certamente completo.

È noto che, se $(A, \mathfrak{m})$ è una coppia henseliana e $\tau$ una topologia lineare (di tipo numerabile) separata sopra $A$, la terna $(A, \mathfrak{m}, \tau)$ non è necessariamente henseliana ([18], n. 6, esempio 2)). Il Teorema 3 ci permette ora di chiarire quali topologie separate $\tau$, introdotte in una coppia henseliana, la rendono una terna henseliana:

CoRollaRIo. - Sia $(A, \mathfrak{m})$ una coppia henseliana $e$ $\tau$ una topologia lineare separata con base numerabile per gli intorni di 0 sopra $A$. Allora condizione necessaria $e$ sufficiente affinchè la terna $(A, \mathfrak{m}, \tau)$ sia henseliana è che $\mathfrak{m}$ sia completo per $\tau$. 
Dimostrazione. - La prima parte dell'enunciato non è altro che l'implicazione 1) $\Rightarrow 2$ ) del teorema 3 . La seconda parte si trova in [18], proposizione 2 .

Vogliamo ora chiarire i precedenti risultati proponendo qualche esempio di terna henseliana $(A, \mathfrak{m}, \tau)$ in cui l'anello $A$ è separato ma non completo; per far questo premettiamo ancora un risultato che ci permette di costruire in modo assai semplice tali esempi ed è conseguenza immediata dei teoremi dati fino ad ora:

Propostzione 2. - Sia $(A, \mathfrak{m}, \tau)$ una terna henseliana tale che $A$ od $\mathfrak{m}$ siano separati e completi per $\tau$. Se $A^{\prime} \dot{e}$ un qualsiasi sottoanello topologico di $A$ contenente $\mathrm{mt}$, allora $\left(A^{\prime}, \mathfrak{m}, \tau^{\prime}\right)$ ̀े una terna henseliana $\left(\tau^{\prime}=\right.$ topologia indotta su $\left.A^{\prime} d a \tau\right)$.

Drmostrazione. - Sia $R$ una serie ristretta a coefficienti in $A^{\prime}$ tale che $\vec{R}=\bar{P} \bar{Q}$ in $\left(A^{\prime} / \mathfrak{m}\right)\{X\}$, con $\bar{P}$ polinomio unitario e $\bar{Q}$ serie ristretta coprimi. La decomposizione si rimonta certo in $A\{X\}: R=P Q$ con $P \rightarrow \bar{P}$ eQ $\rightarrow \bar{Q}$. Poichè $A^{\prime}$ contiene $\mathrm{m}$ è chiaro che $P$ e $Q$ hanno i coeffieienti in $A^{\prime}$.

Si noti che, fra l'altro, la precedente proposizione generalizza lievemente la prima parte della proposizione 3 di [18].

ESEMPI. - Fissiamo una terna henseliana con anello e ideali separati e completi: $A=K[[X, Y]], \mathrm{m}=X K[[X, Y]]$ (ideale generato da $X) \tau=$ topologia $Y$-adica. La terna $(A, \mathfrak{m}, \tau)$ è henseliana per il teorema 3 , in quanto $(A, \mathfrak{m})$ è una coppia henseliana (ottenuta rimpicciolendo l'ideale della coppia henseliana $(K[[X, Y]]$, $X K[[X, Y]]+Y K[[X, Y]]:[11]$, proposizione 4.8) e sia $A$ sia $\mathfrak{m}$ sono separati completi per la topologia $X$-adica. La Proposizione 2 afferma che, se scegliamo un qualsiasi anello $A^{\prime}$ contenente l'ideale generato da $X$ in $A$, la terna $\left(A^{\prime}, \mathfrak{m}, \tau^{\prime}\right)$ $\left(\tau^{\prime}=\right.$ topologia indotta) è henseliana. Possiamo, ad esempio, scegliere $A^{\prime}$ nei seguenti modi:

1) $A^{\prime}=K[Y]_{(P)}+X K[[X, Y]]$

2) $A^{\prime}=Z[Y]+X K[[X, Y]]$ (se $K$ ha caratteristica 0 ); ecc.

Resta quindi chiarito che il teorema 3 descrive effettivamente una classe di terne che non rientrano nel lemma di HENSEL versione BoURBAKI e nemmeno nel teorema 5 di $[18]$.

Concludiamo questo numero esaminando il comportamento di una terna henseliana al variare della topologia. Precisamente vale il seguente

TEorenca 4. - Siano $(A, \mathfrak{m}, \tau)$ una terna henseliana e $\tau^{\prime}$ una topologia lineare separata con base numerabile per gli intorni di 0 , soddisfacente alle seguenti condizioni:

1) $\tau^{\prime} \grave{e}$ più fine di $\tau$;

2) esiste una base di intorni dello 0 di $A$ in $\tau^{\prime}$ formata di chiusi per la topologia $\tau$.

Allora $(A, \mathfrak{m}, \tau)$ è una terna henseliana. 
Dimostrazione. - $(A, \mathfrak{m})$ è una coppia henseliana ed inoltre $\mathfrak{m}$, essendo completo per $\tau$ ([18], proposizione 2), è anche completo per $\tau^{\prime}$ ([8], cap. III, $\S 3$, n. 5, corollario 2 alla proposizione 9). E allora applicabile il Teorema 3 , in base al quale $(A, \mathfrak{m}, \tau)$ ò una terna henseliana.

ESEMPI. - Abbiamo visto che $\left(K[Y]_{(F)}+X K[[X, Y]],(X), \tau_{F}\right)$ è una terna henseliana (esempio precedente). Dal Teorema 4 si deduce che si ottengono ancora terne henseliane sostituendo la topologia $Y$-adica con una delle seguenti:

1) topologia $(X Y)$-adica;

2) topologia con intorni dello 0 costituiti dalla successione di ideali $\left\{X Y^{n}\right\}$.

3. - Nel presente numero ci occupiamo di proprietà algebriche delle terne henseliane e dell'henselizzazione, esaminando quali risultati già validi per le coppie si estendono alle terne.

È noto che, per le coppie, vale il risultato seguente ([11], proposizione 4.8):

"Siano $(A, \mathfrak{m})$ una coppia ed $\mathfrak{n}$ un ideale di A contenuto in $\mathfrak{m}$. Le seguenti condizioni sono equivalenti:

i) $(A, \mathrm{~m})$ è una coppia henseliana;

ii) $(A, \mathfrak{n})$ e $(A / \mathfrak{n}, \mathfrak{m} / \mathfrak{n})$ sono coppie henseliane."

Per generalizzare il precedente risultato alle terne occorre premettere un lemma di carattere topologico:

LEMMA. - Siano A un anello linearmente topologizzato con base numerabile per gli intorni dello 0 ed a un suo ideale chiuso. Allora le seguenti condizioni sono equivalenti:

1) A è separato completo;

2) à è separato completo per la topologia indotta e A/a è separato completo per la topologia quoziente.

\section{Dimostrazione:}

a) 1) $\Rightarrow 2$ ): a è completo perchè chiuso di un completo ([7], cap. II, $\S 3$, n. 4 , proposizione 8). A/a è completo perchè quoziente di un anello completo rispetto ad un ideale chiuso ([9], $\S 3$, n. 1, proposizione 4$)$.

b) 2) $\Rightarrow 1$ ): sia $\hat{A}$ il separato completato di $A$. Allora $\hat{A} / \hat{a}$ è il separato completato di $A / \mathfrak{a}([9], \S 3, \mathrm{n} .1$, corollario 1 alla proposizione 4). Ma $A / \mathfrak{a}$ è separato completo per ipotesi; quindi $A / \mathfrak{a}=\hat{A} / \hat{a}$, il che significa: $\hat{A}=A+\hat{\mathfrak{a}}=A+\mathfrak{a}=A$.

Propostzrone 3. - Siano $(A, \mathfrak{m}, \tau)$ una terna con una base numerabile per gli intorni dello 0 ed $\mathfrak{n}$ un ideale chiuso contenuto in $\mathrm{m}$. Allora le seguenti condizioni sono equi- 
valenti:

1) $(A, \mathfrak{H}, \tau)$ è una terna henseliana;

2) $(A, \mathfrak{n}, \tau)$ e $\left(A / \mathfrak{n}, \mathfrak{m} / \mathfrak{n}, \tau_{\mathfrak{n}}\right)$ sono terne henseliane $\left(\tau_{\mathfrak{n}}=\right.$ topologia quoziente $)$.

\section{DMrostrazione:}

a) 1) $\Rightarrow 2)$ : poichè $(A, \mathfrak{m}, \tau)$ è una terna henseliana, $\mathfrak{m}$ è separato completo ([18], proposizione 2). Di qui segue che $\mathfrak{n}$ è pure separato completo, come sottospazio chiuso di uno spazio uniforme completo ([7], cap. II, $\$ 3$, n. 4, proposizione 8). Poichè inoltre $(A, \mathfrak{n})$ è una coppia henseliana ([11], proposizione 4.8$),(A, \mathfrak{n}, \tau)$ è una terna henseliana per il corollario al teorema 3.

Per la seconda parte dell'enunciato basta osservare che $(A / \mathfrak{t}, \mathfrak{n t} / \mathfrak{H})$ è una coppia henseliana ([11], proposizione 4.8) e che $\mathfrak{m} / \mathfrak{n}$ è separato completo $([9], \S 3, \mathfrak{n} .1$, proposizione 4); si può quindi applicare il corollario al teorema 3.

b) 2) $\Rightarrow 1):(A, \mathfrak{m})$ è una coppia henseliana ([11], proposizione 4.8$)$ ed $\mathfrak{m}$ è separato completo in quanto lo sono $\mathfrak{n}$ ed $\mathfrak{m} / \mathfrak{n}$ ([18], proposizione 2 ), per il precedente lemma; si può ancora applicare il corollario al teorema 3.

OSSERVAzione. - Eliminando l'ipotesi di numerabilità nel teorema 7, cade la dimostrazione ora fatta dell'implicazione 1) $\Rightarrow 2$ ), a meno che non si facciano ulteriori ipotesi di completezza, per esempio supponendo che $A$ ed $A / \mathfrak{n}$ siano separati completi. In questo caso l'implicazione 1) $\Rightarrow 2$ ) segue dal teorema 5 di [18].

Per quanto riguarda invece l'implicazione 2$) \Rightarrow 1$ ) è possibile dare una dimostrazione che non coinvolge la numerabilità.

In effetti, sia $R$ una serie ristretta sopra $A$ tale che $\bar{R}=\bar{P} \bar{Q}$ in $(A / \mathrm{m})\{X\}$, essendo $\bar{P}$ e $\bar{Q}$ un polinomio unitario e una serie ristretta coprimi. Identificando $A / \mathfrak{m}$ con $(A / \mathfrak{n}) /(\mathfrak{m} / \mathfrak{n})([8]$, cap. III, $\S 2$, n. 7 , corollario alla proposizione 22$)$, possiamo leggere $\bar{R}, \bar{P}$ e $\bar{Q}$ in $((A / \mathfrak{n}) /(\mathfrak{m} / \mathfrak{n}))\{X\}$. Sia ora $R^{\prime}$ l'immagine canonica di $R$ in $(A / \mathfrak{n})\{X\}$; allora esiste una e una sola coppia $\left(P^{\prime}, Q^{\prime}\right)$ formata da un polinomio unitario $P^{\prime}$ e da una serie ristretta $Q^{\prime}$ coprimi in $(A / \mathfrak{n})\{X\}$ tale che $R^{\prime}=P^{\prime} Q^{\prime}$, $P \rightarrow P^{\prime}, Q \rightarrow Q^{\prime}$. Poichè $(A, \mathfrak{n}, \tau)$ è una terna henseliana, esiste una e una sola coppia $(P, Q)$ formata da un polinomio unitario $P$ e da una serie ristretta $Q$ coprimi in $A\{X\}$ tale che $R=P Q$ ed inoltre $P \rightarrow P^{\prime}, Q \rightarrow Q^{\prime}$. E chiaro che $(P, Q)$ è il rimontamento cercato.

Per le coppie è ancora noto il seguente risultato ([10], teorema 4.6):

"Se $(A, \mathfrak{m})$ è una coppia henseliana e $A^{\prime}$ è una A-algebra intera sopra $A$, allora è henseliana anehe la coppia $\left(A^{\prime}, \mathfrak{m} A^{\prime}\right)$ ".

E ovvia la seguente traduzione al caso delle terne:

Propostzione 4. - Siano $(A, \mathfrak{m}, \tau)$ una terna henseliana e $\left(A^{\prime}, \mathfrak{m}^{\prime}, \tau^{\prime}\right)$ una terna tale che siano soddisfatte le seguenti proprietà: 
1) $A^{\prime}$ è una A-algebra intera sopra $A$;

2) $\mathfrak{m}^{\prime}=\mathfrak{m} A^{\prime}$;

3) $\mathfrak{m}^{\prime}$ è separato completo rispetto $a \tau^{\prime}$.

Allora $\left(A^{\prime}, \mathfrak{m}^{\prime}, \tau^{\prime}\right)$ è una terna henseliana.

Dimostraztone. - $\left(A^{\prime}, \mathfrak{m}^{\prime}\right)$ è una coppia henseliana e quindi è applicabile il corollario al teorema 3 .

Fra $i$ casi interessanti che rientrano nella proposizione 4 si ha il seguente

Corollario. - Siano $(A, \mathfrak{m}, \tau)$ una terna henseliana e a un ideale chiuso di $A$ tale che $\mathrm{m}(A / \mathfrak{a})$ sia separato completo per la topologia quoziente. Allora la terna $(A / \mathfrak{a}$, $\left.\mathfrak{m}(A / \mathfrak{a}), \tau_{\mathfrak{a}}\right)$ è henseliana $\left(\tau_{\mathfrak{a}}=\right.$ topologia quoziente).

E infine noto che, nel caso delle coppie, l'henselizzazione commuta con il passaggio al quoziente ([18], corollario 1 al teorema 1 ).

Per le terne possiamo dare un risultato lievemente più restrittivo:

Propostzione 5. - Siano $(A, \mathfrak{m}, \tau)$ una terna con base numerabile per gli intorni dello 0 di $A$ e a un ideale chiuso contenuto in $\mathrm{m}$. Siano poi $\left({ }^{n} A,{ }^{n} \mathrm{~m}\right)$ l'henselizzazione della coppia $(A, \mathfrak{m})$ e ${ }^{h} \tau$ la topologia estensione di $\tau$ ad ${ }^{h} A$. Sia infine $\overline{\mathfrak{a}}=\left({ }^{h} \mathfrak{a}\right)^{\wedge}$ il separato completato di ${ }^{n} \mathfrak{a}=\mathfrak{a}^{h} A$ rispetto ad ${ }^{h} \tau$. Allora, detta $\left(A^{\prime}, \mathfrak{m}^{\prime}, \tau^{\prime}\right)$ l'henselizzazione della terna $(A, \mathfrak{m}, \tau)$, l'henselizzazione della terna $\left(A / \mathfrak{a}, \mathfrak{m} / \mathfrak{a}, \tau_{\mathfrak{a}}\right)\left(\tau_{\mathfrak{a}}=\right.$ topologia quoziente) coincide con la seguente terna: $\left(A^{\prime} / \sqrt[\mathfrak{a}]{,} \mathfrak{m}^{\prime} / \mathfrak{a}, \tau_{\overline{\mathfrak{a}}}^{\prime}\right)\left(\tau_{\overline{\mathfrak{a}}}^{\prime}=\right.$ topologia quoziente $)$.

Drmostrazione. - Cominciamo coll'osservare che, essendo a contenuto in $\mathfrak{m}$, ${ }^{n} \mathfrak{a}$ è contenuto in ${ }^{h} \mathfrak{m}$, quindi $\overline{\mathfrak{a}}=\left({ }^{h} \mathfrak{a}\right)^{\wedge}$ è contenuto in $\mathfrak{m}^{\prime}=\left({ }^{h} \mathfrak{m}\right)^{\wedge}([18]$, teorema 7$)$. Perciò $\overline{\mathfrak{a}}$ è contenuto in $A^{\prime}$.

La terna $\left(A^{\prime} \sqrt{a}, \mathfrak{m}^{\prime} / \bar{a}, \tau_{\overline{\mathfrak{a}}}^{\prime}\right)$ è henseliana per la proposizione 3 . Verifichiamo che è soluzione del problema universale che definisce la henselizzazione di $(A, \mathfrak{m}, \tau)$.

Sia percio $\varphi_{\mathfrak{a}}:\left(A / \mathfrak{a}, \mathfrak{m} / \mathfrak{a}, \tau_{\mathfrak{a}}\right) \rightarrow(C, \mathfrak{n}, \delta)$ un morfismo in una terna henseliana. Detta $\pi: A \rightarrow A / a$ la proiezione canonica di $A$ sul quoziente ed $f$ l'immersione di $A$ nella sua henselizzazione $A^{\prime}$, resta individuato uno ed un solo morfismo di terne $\varphi^{\prime}:\left(A^{\prime}, \mathfrak{m}^{\prime}, \tau^{\prime}\right) \rightarrow(0, \mathfrak{n}, \delta)$ tale che si abbia: $\varphi^{\prime} \circ f=\varphi_{\mathfrak{a}} \circ \pi$. Il morfismo $\varphi_{\mathfrak{a}} \circ \pi$ si annulla ovviamente sopra $a$. Non è difficile verificare che di qui segue che $\varphi^{\prime}$ si annulla sopra $\vec{a}$ (basta ricordare come è definito $\varphi^{\prime}:[18]$, corollario al teorema 6 e teorema 7 ). È perciò definito un morfismo della terna $\left(A^{\prime} / \overline{\mathfrak{a}}, \mathfrak{m}^{\prime} / \mathfrak{a}, \tau_{\overline{\mathfrak{a}}}^{\prime}\right)$ nella terna $(C, \mathfrak{n}, \delta)$ che rende commutativo il seguente diagramma:

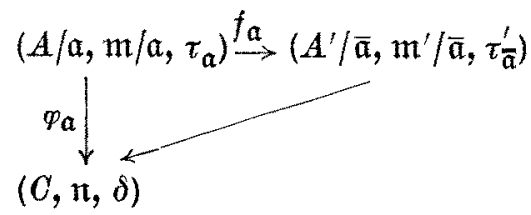

dove $f_{\mathfrak{a}}$ è il morfismo che si deduce per passaggio al quoziente da $f$.

E evidente che il morfismo ora indicato è unico, per l'unicità di $\varphi^{\prime}$. 
OSSERVAzIONE. - Segue immediatamente dalla nostra dimostrazione il fatto, non ovvio a priori, che $A / \mathfrak{a}$ è un sottoanello topologico di $A^{\prime} / \bar{a}$, in quanto una terna con ideale contenuto nel radicale è immersa algebricamente e topologicamente nella sua henselizzazione: [18], teorema 7 .

\section{BIBLIOGRAFIA}

[1] G. Azumax A, On Maximally central Algebras, Nagoya Math. J., t. 2, (1951), pp. 119-150.

[2]. N. Bourbaki, Algebre, cap. Ir, Hermann, Paris (1967).

[3] N. BounbaKr, Algèbre, cap. VIII, Hermann, Paris (1961).

[4] N. Bourbaki, Algèbre Commutative, capp. I, II, Hermann, Paris (1965).

[5] N. Bourbati, Algèbre Commutative, capp. III, IV, Hermann, Paris (1961).

[6] N. Bounbari, Théorie des Ensembles, cap. III, Hermann, Paris (1967).

[7] N. BourbaK, Topologie Générale, capp. I, II, Hermann, Paris (1965).

[8] N. Bourbakr, Topologie Générale, capp. III, IV, Hermann, Paris (1960).

[9] N. Bourbaki, Topologie Générale, cap. IX, Hermann, Paris (1958).

[10] S. GRECO, Algebras over nonlocal Hensel rings, Journal of Algebra, 8 (1968), pp. 45-59.

[11] S. GRECo, Henselization of a ring with respect to an ideal, Trans. Amer. Math. Soc., 144 (1969), pp. $43-65$.

[12] S. GRECo, Sugli omomorismi piatti e non ramificati, Estratto da "Le Matematiche", 24, fasc. 2 (1969).

[13] J. P. Lafon, Anneaux Henseliens, Bull. Soc. Math. France, 91 (1963), pp. 77-108.

[14] M. NaGata, On the theory of henselian rings: I) Nagoya Math. J., t. 5 (1953), pp. 45-57; II) id., t. 7 (1954), pp. 1-19; III) Mem. Coll. Se. Univ. Kyoto, serie A, Math., t. 32 (1960), pp. $93-101$.

[15] M. NAGata, Local Rings, Interscience, New York (1962).

[16] M. RaYNaUd, Anneaux Locaux Henséliens, Springer-Verlag, New York (1970).

[17] P. SALMon, Sur les séries formelles restreintes, Bull. Soc. Math. France, 92 (1964), pp. 385-410.

[18] P. Valabrega, Anelli henseliani topologici, Amali di Matematica pura e applicata, 4 (1972), pp. $283-303$. 